\title{
Impact of Front Line Demostration (FLD) on the Yield and Economics of Small Millet on Bastar District of Chhattisgarh, India
}

\author{
Ashwani Kumar Thakur*, Prafull Kumar and Subhas Chandra Yadav \\ SG College of Agriculture and Research Station, Jagdalpur (CG) - 494005, India \\ *Corresponding author
}

\begin{tabular}{|l|l|}
\hline \multicolumn{1}{l|}{ A B S T R A C T } \\
\cline { 2 - 2 } $\begin{array}{l}\text { Keywords } \\
\begin{array}{l}\text { Front line } \\
\text { Smonstration, } \\
\text { Bastar plateau zone. }\end{array}\end{array}$ & $\begin{array}{l}\text { Millet has emerged as one of the most suitable alternative to minor millet } \\
\text { and minor pulses in Bastar plateau zone of Bastar district at Chhattisgarh. } \\
\text { However, the productivity of small millet in the Bastar plateau zone is very } \\
\text { low. One of the major constraints of traditional millet farming is low } \\
\text { productivity due to lack of recommended package of practices and high } \\
\text { yielding varieties. To replace this inconsistency of practices, SG College of } \\
\text { Agriculture and Research under AICRPSM, Jagdalpur conducted } \\
\text { experiments in the farmer's field. Through frontline demonstration farmers } \\
\text { were provided with all the basic inputs (improved practices). This resulted } \\
\text { in higher yield than that obtained through the farmer's practices. } \\
\text { 19 July 2017 } \\
\text { Available Online: } \\
\text { 10 September 2017 }\end{array}$ \\
\hline \hline
\end{tabular}

\section{Introduction}

Minor millets categorized as coarse cereals are staple food for the tribal people where cultivation of major cereals like rice, wheat and maize is either not popular or fail to produce substantial yield (Amadoul et al., 2013). India is considered as hub for these minor crops, according to the latest data, the world total production of millet grains at last count was 762,712 metric tonnes and the top producer was India with an annual production of 334,500 tonnes contributing $43.85 \%$ (http://faostat.fao.org/site/339/default.aspx).

Finger millet (Eleucine coracana (L.) Gaertn.) is a stable cereal food crop for millions of people in the semi-arid region of the world, particularly in Africa and India, and especially those who live by subsistence farming. This crop is cultivated in a wide geographical zone ranging from Senegal, Niger, Nigeria, across eastern and southern Africa, through the Middle East and into tropical Asia (Anonyms, 1996 and Burkill, 1985). Finger millet [Eleusine coracana (L.) Gaertn.] is among the most cultivated millets and belongs to the genus Eleusine, in the Chloridoidae subfamily (Clayton and Renvoze, 1986).

It is a native African crop which is also extremely important in South Asia (India and Nepal) (National Research Council, 1996). It has a 97 to $99 \%$ self-pollinating (Hilu and de Wet, 1980) and takes between 2.5 to 6 months to mature (Watson and Dallwitz, 1992). The 
crop is adapted to a wide range of environments and can be grown in a variety of soils with medium or low water holding capacity (National Research Council, 1996), but requires rainfall of at least $800 \mathrm{~mm}$ per annum (Van Wyk and Gericke, 2000). In Africa and South Asia, finger millet is a staple food grain upon which millions depend however, finger millet straw also makes good animal fodder, containing up to $60 \%$ total digestible nutrients (National Research Council, 1996). There is a growing realization that millets, including finger millet would produce a more dependable harvest compared to other crops especially under marginal and sub marginal conditions of soil fertility and limited moisture (Seetharam, 1986).

In Bastar district of Chhattisgarh, which is one of the largest congregations of tribal population $(67.4 \%)$, inhabitants mainly grow millets on upland situations and form major component of their daily food consumption. In addition to be nutritionally rich, the advantage of growing minor millets is that it is a rainfed crop which forms part of a multicropping system, in the sense that it is mostly grown along with legumes and oilseeds (Chopra and Neelam 2004; Pradhan et al., 2010) On the darker side, these are underutilized and neglected crops owing to their lower preference driven by affluence, longer time and efforts involved in processing of the millets and the lower cooking quality. If these problems could be solved, their high nutritional value can make them doubly valuable as food for farming families and a potential source of income.

\section{Materials and Methods}

The present study was carried out by the SG college of Agriculture and Research Station under All India Co- ordinated Research Project on small millets (AICRPSM), Jagdalpur, Chhattisgarh during Kharif season during 2015. In finger millet cv. GPU28, nine farmers, in kodo millet cv. JK 439, 10 farmers and little millet $\mathrm{cv}$. JK 8, five farmers were selected in Bastar and Dantewada district of Bastar Plateau Zone of Chhattisgarh. Total 24 front line demonstrations were carried out in an area 26 ha in different villages. The studies were under taken through farmers meeting, survey and diagnostic visit for selection of the farmers and villages. Orientation training programme was imparted to the beneficiaries related to crop before conducting demonstration. Farmers were getting lower yield due to local varieties, imbalanced fertilizer, broadcasting of seed, plant protection practices were not adopted and poor weed management. In improved package of practices, good quality seed, recommended balanced fertilizer, line sowing and timely sowing, effective plant protection and chemical and manual weed management and frequent monitoring the farmer's field during cropping season (Table 1). Yield data were collected from farmer's practices and improved practices. Cost of cultivation, gross return, net return and benefit cost ratio $(\mathrm{B}$ : $\mathrm{C}$ ratio) were computed and analysed. The technology gap and technology index were calculated using the following formula as given by Samui et al., (2000).

Technology index $=$ Potential Yield Demonstration Yield/ Potential Yield x 100

Technology gap = Potential Yield Demonstration Yield

Extension gap $=$ Demonstration yield - Yield under Farmers Practices

B: $\mathrm{C}$ ratio $=$ Net income $($ Rs. $/$ ha $) /$ cost of cultivation (Rs. / ha)

$\%$ increased over farmers practices $=$ Improved practices - Farmers practices / farmers practices x 100 


\section{Results and Discussion}

\section{Yield}

The average yield of millets under improved practices, $15.09 \mathrm{q} \mathrm{ha}^{-1}$ in finger millet, $14.57 \mathrm{q}$ $\mathrm{ha}^{-1}$ in kodo millet and $5.85 \mathrm{q} \mathrm{ha}^{-1}$ in little millet was much higher than as compared to average yield of farmer's practices $(6.33,6.29$ and $2.75 \mathrm{q} \mathrm{ha}^{-1}$ in finger, kodo and little millet) (Table 2). The average percentage increased in the yield over farmer's practices was $140.12 \%, 148.64 \%$ and $115.64 \%$ in finger, kodo and little millet. The results indicated that the FLD (Tables 3 and 4) gives a better impact over the farming family of Bastar and Dantewada districts as they were encouraged by the new agricultural technology applied in the improved practices (Table 5). This finding is also observed by
Tiwari and Saxena (2001), Dudhade et al., (2009), Poonia and Pithia (2010), Poonia and Pithia (2011), Painkra et al., (2012) and Raj et al., (2013).

\section{Extension gap}

The average extension gap in the improved practices was $8.76 \mathrm{q} \mathrm{ha}^{-1}$ in finger millet, 8.28 $\mathrm{q} \mathrm{ha}^{-1}$ in kodo millet and $3.11 \mathrm{q} \mathrm{ha}^{-1}$ in little millet. This gap shows that there is need to educate the farmers community by various adoption methods like improved agricultural production techniques. Subsequent change the low yielding local or old varieties and improved management practices is needed to increase the yield per capita and overcome the extension gap (Table 5). The findings of the present study are in line with the findings of Hiremath and Nagraju (2010).

Table.1 Particular showing the details of maize growing under Improved practices and farmers practices

\begin{tabular}{|c|c|c|c|}
\hline S. No. & Operations & Farmer practices & Improved practices \\
\hline 01 & $\begin{array}{l}\text { Seed material } \\
\text { used }\end{array}$ & $\begin{array}{l}\text { Local variety or } \\
\text { composite variety }\end{array}$ & Improved varieties \\
\hline 02 & Sowing method & Broad casting & Line sowing \\
\hline 03 & $\begin{array}{l}\text { Fertilizer } \\
\text { application }\end{array}$ & $\begin{array}{l}\text { 00:00:00 NPK (kg } \\
\left.\mathrm{ha}^{-1}\right)\end{array}$ & $\begin{array}{l}\text { 50:40:20 and 20:20:10 NPK }\left(\mathrm{kg} \mathrm{ha}^{-1}\right) \text { for } \\
\text { finger millet, and kodo and little millet. } \\
\text { Applied half dose of } \mathrm{N} \text { as basal and half at } \\
\text { tillering stage. Full dose of } \mathrm{P} \text { and } \mathrm{K} \text { was } \\
\text { applied at the time of sowing. }\end{array}$ \\
\hline 04 & $\begin{array}{l}\text { Weed } \\
\text { management }\end{array}$ & No weeding & $\begin{array}{l}\text { Chemical weed control, Isoproturon @ } 0.5 \mathrm{~kg} \\
\text { ai ha }{ }^{-1} \text { as pre- emergence and need based } \\
\text { weeding }\end{array}$ \\
\hline 05 & $\begin{array}{l}\text { Stem borer and } \\
\text { aphid }\end{array}$ & $\begin{array}{l}\text { No any control } \\
\text { measures }\end{array}$ & $\begin{array}{l}\text { Chlorpyriphos@1.5 ml per litre of water } \\
\text { and imidacloprid }\end{array}$ \\
\hline
\end{tabular}


Table.2 Effect of FLDs at different farmers with improved practices (IP) and farmers practices (FP), percent increase over farmer's practices and economics in Finger millet

\begin{tabular}{|c|c|c|c|c|c|c|c|c|c|c|c|c|c|c|c|}
\hline \multicolumn{16}{|c|}{ Finger Millet } \\
\hline $\begin{array}{c}\text { S } \\
\text { No. }\end{array}$ & $\begin{array}{l}\text { Name of } \\
\text { Farmer }\end{array}$ & $\begin{array}{l}\text { Cate } \\
\text { gory }\end{array}$ & $\begin{array}{c}\text { Villag } \\
\text { e }\end{array}$ & $\begin{array}{l}\text { Dist/ } \\
\text { Block }\end{array}$ & $\begin{array}{c}\text { Variet } \\
\mathbf{y}\end{array}$ & Crop & $\begin{array}{c}\text { Are } \\
\mathbf{a} \\
(\mathbf{h a})\end{array}$ & $\begin{array}{c}\text { IP } \\
\text { Yield } \\
\left(q_{1} \text { ha }^{-}\right.\end{array}$ & $\begin{array}{c}\text { FP } \\
\text { Yield } \\
\left(\mathbf{q ~ h a}^{-1}\right)\end{array}$ & $\begin{array}{c}\text { Net } \\
\text { Income } \\
(₹ \text { ha-1) }\end{array}$ & $\begin{array}{l}\text { B:C } \\
\text { Ratio }\end{array}$ & TI & TG & EG & IOFP \\
\hline 1 & $\begin{array}{l}\text { Surjo/Lak } \\
\text { hmu }\end{array}$ & ST & $\begin{array}{l}\text { Kola } \\
\text { wal }\end{array}$ & $\begin{array}{c}\text { Bastar / } \\
\text { Bakawand }\end{array}$ & $\begin{array}{c}\text { GPU- } \\
28\end{array}$ & $\begin{array}{l}\text { Finger } \\
\text { millet }\end{array}$ & 1.00 & 14.90 & 6.10 & 13865.00 & 1.63 & 40.4 & 10.1 & 8.8 & 144.26 \\
\hline 3 & $\begin{array}{l}\text { Sampat/B } \\
\text { aijnath }\end{array}$ & ST & $\begin{array}{l}\text { Kola } \\
\text { wal }\end{array}$ & $\begin{array}{c}\text { Bastar / } \\
\text { Bakawand }\end{array}$ & $\begin{array}{c}\text { GPU- } \\
28\end{array}$ & $\begin{array}{l}\text { Finger } \\
\text { millet }\end{array}$ & 1.50 & 17.80 & 6.10 & 18215.00 & 2.15 & 28.8 & 7.2 & 11.7 & 191.80 \\
\hline 4 & $\begin{array}{l}\text { Kurso/Ja } \\
\text { gbandhu }\end{array}$ & ST & $\begin{array}{l}\text { Kumh } \\
\text { rawan } \\
\text { d }\end{array}$ & $\begin{array}{c}\text { Bastar / } \\
\text { Bakawand }\end{array}$ & $\begin{array}{c}\text { GPU- } \\
28\end{array}$ & $\begin{array}{l}\text { Finger } \\
\text { millet }\end{array}$ & 1.00 & 13.90 & 6.85 & 12365.00 & 1.46 & 44.4 & 11.1 & 7.05 & 102.92 \\
\hline 6 & $\begin{array}{l}\text { Dumar/R } \\
\text { ameshwa } \\
r\end{array}$ & ST & $\begin{array}{l}\text { Kola } \\
\text { wal }\end{array}$ & $\begin{array}{c}\text { Bastar / } \\
\text { Bakawand }\end{array}$ & $\begin{array}{c}\text { GPU- } \\
28\end{array}$ & $\begin{array}{l}\text { Finger } \\
\text { millet }\end{array}$ & 1.00 & 17.30 & 7.65 & 17465.00 & 2.06 & 30.8 & 7.7 & 9.65 & 126.14 \\
\hline 7 & $\begin{array}{l}\text { Fulchand/ } \\
\text { Chaitan }\end{array}$ & $\begin{array}{c}\text { OB } \\
\mathrm{C}\end{array}$ & $\begin{array}{l}\text { Kumh } \\
\text { rawan } \\
\text { d }\end{array}$ & $\begin{array}{c}\text { Bastar / } \\
\text { Bakawand }\end{array}$ & $\begin{array}{c}\text { GPU- } \\
28\end{array}$ & $\begin{array}{l}\text { Finger } \\
\text { millet }\end{array}$ & 1.00 & 14.20 & 6.85 & 12815.00 & 1.51 & 43.2 & 10.8 & 7.35 & 107.30 \\
\hline 8 & $\begin{array}{l}\text { Mosu/Gu } \\
\text { dru }\end{array}$ & ST & $\begin{array}{l}\text { Basta } \\
\text { nar }\end{array}$ & $\begin{array}{c}\text { Bastanar/ } \\
\text { Dantewada }\end{array}$ & $\begin{array}{c}\text { GPU- } \\
28\end{array}$ & $\begin{array}{l}\text { Finger } \\
\text { millet }\end{array}$ & 1.50 & 12.60 & 5.25 & 10415.00 & 1.23 & 49.6 & 12.4 & 7.35 & 140.00 \\
\hline 9 & $\begin{array}{l}\text { Bakanu/ } \\
\text { Denga }\end{array}$ & ST & $\begin{array}{l}\text { Basta } \\
\text { nar }\end{array}$ & $\begin{array}{l}\text { Bastanar/ } \\
\text { Dantewada }\end{array}$ & $\begin{array}{c}\text { GPU- } \\
28\end{array}$ & $\begin{array}{l}\text { Finger } \\
\text { millet }\end{array}$ & 1.00 & 13.56 & 5.25 & 11855.00 & 1.40 & 45.76 & 11.44 & 8.31 & 158.29 \\
\hline
\end{tabular}


Table.3 Effect of FLDs at different farmers with improved practices (IP) and farmers practices (FP), percent increase over farmer's practices and economics in Kodo millet

\begin{tabular}{|c|c|c|c|c|c|c|c|c|c|c|c|c|c|c|c|}
\hline \multicolumn{16}{|c|}{ Kodo millet } \\
\hline $\begin{array}{c}\text { S } \\
\text { No. }\end{array}$ & $\begin{array}{l}\text { Name of } \\
\text { Farmer }\end{array}$ & $\begin{array}{c}\text { Categ } \\
\text { ory }\end{array}$ & Village & $\begin{array}{l}\text { Dist/ } \\
\text { Block }\end{array}$ & $\begin{array}{c}\text { Variet } \\
\mathbf{y}\end{array}$ & Crop & $\begin{array}{c}\text { Area } \\
\text { (ha) }\end{array}$ & $\begin{array}{c}\text { IP } \\
\text { Yield } \\
\left(\mathbf{q} \mathbf{h a}^{-1}\right)\end{array}$ & $\begin{array}{c}\text { FP } \\
\text { Yield } \\
\left(\mathbf{q} \mathbf{h a}^{-1}\right)\end{array}$ & $\begin{array}{c}\text { Net } \\
\text { Income } \\
\text { (₹ ha-1) }\end{array}$ & $\begin{array}{c}\text { B:C } \\
\text { Ratio }\end{array}$ & TI & TG & EG & IOFP \\
\hline 1 & $\begin{array}{l}\text { Santuram/R } \\
\text { updhar }\end{array}$ & $\mathrm{ST}$ & Kolawal & $\begin{array}{c}\text { Bastar / } \\
\text { Bakawand }\end{array}$ & JK 439 & $\begin{array}{l}\text { Kodo } \\
\text { millet }\end{array}$ & 1.50 & 14.90 & 7.65 & 21067.00 & 3.24 & 25.50 & 5.10 & 7.25 & 94.77 \\
\hline 2 & $\begin{array}{l}\text { Puru/Sukhd } \\
\text { ev }\end{array}$ & ST & Kolawal & $\begin{array}{c}\text { Bastar / } \\
\text { Bakawand }\end{array}$ & JK 439 & $\begin{array}{l}\text { Kodo } \\
\text { millet }\end{array}$ & 1.60 & 15.20 & 7.65 & 21622.00 & 3.33 & 24.00 & 4.80 & 7.55 & 98.69 \\
\hline 3 & $\begin{array}{l}\text { Chandru/So } \\
\text { nu }\end{array}$ & ST & $\begin{array}{l}\text { Kumhra } \\
\text { wand }\end{array}$ & $\begin{array}{c}\text { Bastar / } \\
\text { Bakawand }\end{array}$ & JK 439 & $\begin{array}{l}\text { Kodo } \\
\text { millet }\end{array}$ & 0.50 & 14.56 & 7.65 & 20438.00 & 3.15 & 27.20 & 5.44 & 6.91 & 90.33 \\
\hline 4 & $\begin{array}{l}\text { Chain/Pura } \\
\mathrm{n}\end{array}$ & $\mathrm{ST}$ & $\begin{array}{l}\text { Kumhra } \\
\text { wand }\end{array}$ & $\begin{array}{c}\text { Bastar / } \\
\text { Bakawand }\end{array}$ & JK 439 & $\begin{array}{l}\text { Kodo } \\
\text { millet }\end{array}$ & 1.00 & 14.85 & 7.65 & 20974.50 & 3.23 & 25.75 & 5.15 & 7.20 & 94.12 \\
\hline 5 & $\begin{array}{l}\text { Baman/Pan } \\
\text { du }\end{array}$ & $\mathrm{ST}$ & Bastanar & $\begin{array}{c}\text { Bastanar/ } \\
\text { Dantewada }\end{array}$ & JK 439 & $\begin{array}{l}\text { Kodo } \\
\text { millet }\end{array}$ & 1.50 & 13.60 & 4.25 & 18662.00 & 2.87 & 32.00 & 6.40 & 9.35 & 220.00 \\
\hline 6 & $\begin{array}{l}\text { Lakhmu/Ma } \\
\text { sa }\end{array}$ & St & Bastanar & $\begin{array}{c}\text { Bastanar/ } \\
\text { Dantewada }\end{array}$ & JK 439 & $\begin{array}{l}\text { Kodo } \\
\text { millet }\end{array}$ & 0.50 & 14.00 & 4.25 & 19402.00 & 2.99 & 30.00 & 6.00 & 9.75 & 229.41 \\
\hline 7 & $\begin{array}{l}\text { Sonadhar/Ja } \\
\text { gbandhu }\end{array}$ & ST & $\begin{array}{l}\text { Kumhra } \\
\text { wand }\end{array}$ & $\begin{array}{c}\text { Bastanar/ } \\
\text { Dantewada }\end{array}$ & JK 439 & $\begin{array}{l}\text { Kodo } \\
\text { millet }\end{array}$ & 0.50 & 14.56 & 7.65 & 20438.00 & 3.15 & 27.20 & 5.44 & 6.91 & 90.33 \\
\hline 8 & $\begin{array}{l}\text { Arjun/Jagba } \\
\text { ndhu }\end{array}$ & ST & $\begin{array}{l}\text { Kumhra } \\
\text { wand }\end{array}$ & $\begin{array}{c}\text { Bastanar/ } \\
\text { Dantewada }\end{array}$ & JK 439 & $\begin{array}{l}\text { Kodo } \\
\text { millet }\end{array}$ & 0.50 & 15.90 & 7.65 & 22917.00 & 3.53 & 20.50 & 4.10 & 8.25 & 107.84 \\
\hline 9 & Talo/Mosu & ST & Bastanar & $\begin{array}{c}\text { Bastanar/ } \\
\text { Dantewada }\end{array}$ & JK 439 & $\begin{array}{l}\text { Kodo } \\
\text { millet }\end{array}$ & 1.00 & 13.89 & 4.25 & 19198.50 & 2.95 & 30.55 & 6.11 & 9.64 & 226.82 \\
\hline 10 & $\begin{array}{l}\text { Chhadmo/S } \\
\text { ukro }\end{array}$ & ST & Bodenar & $\begin{array}{c}\text { Bastanar/ } \\
\text { Dantewada }\end{array}$ & JK 439 & $\begin{array}{l}\text { Kodo } \\
\text { millet }\end{array}$ & 1.50 & 14.20 & 4.25 & 19772.00 & 3.04 & 29.00 & 5.80 & 9.95 & 234.12 \\
\hline
\end{tabular}


Table.4 Effect of FLDs at different farmers with improved practices (IP) and farmers practices (FP), percent increase over farmer's practices and economics in little millet

\begin{tabular}{|c|c|c|c|c|c|c|c|c|c|c|c|c|c|c|c|}
\hline \multicolumn{16}{|c|}{ Little millet } \\
\hline $\begin{array}{c}\text { S } \\
\text { No. }\end{array}$ & $\begin{array}{l}\text { Name of } \\
\text { Farmer }\end{array}$ & $\begin{array}{l}\text { Cate } \\
\text { gory }\end{array}$ & Village & $\begin{array}{l}\text { Dist/ } \\
\text { Block }\end{array}$ & $\begin{array}{l}\text { Vari } \\
\text { ety }\end{array}$ & Crop & $\begin{array}{c}\text { Area } \\
\text { (ha) }\end{array}$ & $\begin{array}{c}\text { IP } \\
\text { Yield } \\
\left(\mathbf{q} \text { ha }^{-1}\right)\end{array}$ & $\begin{array}{c}\text { FP } \\
\text { Yield } \\
\left(\mathbf{q} \mathbf{h a}^{-1}\right)\end{array}$ & $\begin{array}{c}\text { Net } \\
\text { Income } \\
\left(₹ \mathbf{h a}^{-1}\right)\end{array}$ & $\begin{array}{l}\text { B:C } \\
\text { Ratio }\end{array}$ & TI & TG & EG & IOFP \\
\hline 1 & $\begin{array}{l}\text { Chhirma/ } \\
\text { Pandu }\end{array}$ & $\mathrm{ST}$ & Bastanar & $\begin{array}{c}\text { Bastanar/ } \\
\text { Dantewada }\end{array}$ & JK 8 & $\begin{array}{l}\text { Little } \\
\text { millet }\end{array}$ & 1.50 & 5.60 & 2.50 & $\begin{array}{c}3360.0 \\
0\end{array}$ & 0.60 & 78.57 & 4.40 & 3.10 & 124.00 \\
\hline 2 & $\begin{array}{l}\text { Chetan/P } \\
\text { aklu }\end{array}$ & ST & Bodenar & $\begin{array}{c}\text { Bastanar/ } \\
\text { Dantewada }\end{array}$ & JK 8 & $\begin{array}{l}\text { Little } \\
\text { millet }\end{array}$ & 1.00 & 5.90 & 3.12 & $\begin{array}{c}3840.0 \\
0\end{array}$ & 0.69 & 69.49 & 4.10 & 2.78 & 89.10 \\
\hline 3 & $\begin{array}{l}\text { Balram/S } \\
\text { ethi }\end{array}$ & ST & Bodenar & $\begin{array}{c}\text { Bastanar/ } \\
\text { Dantewada }\end{array}$ & JK 8 & $\begin{array}{l}\text { Little } \\
\text { millet }\end{array}$ & 1.50 & 5.75 & 3.12 & $\begin{array}{c}3600.0 \\
0 \\
\end{array}$ & 0.64 & 73.91 & 4.25 & 2.63 & 84.29 \\
\hline 4 & $\begin{array}{l}\text { Pandu/L } \\
\text { achhu }\end{array}$ & ST & Mutanpal & $\begin{array}{c}\text { Bastanar/ } \\
\text { Dantewada }\end{array}$ & JK 8 & $\begin{array}{l}\text { Little } \\
\text { millet }\end{array}$ & 0.50 & 6.12 & 2.50 & $\begin{array}{c}4192.0 \\
0\end{array}$ & 0.75 & 63.40 & 3.88 & 3.62 & 144.80 \\
\hline 5 & $\begin{array}{l}\text { Rukmani } \\
\text { /Bijo }\end{array}$ & $\mathrm{ST}$ & Kilepal & $\begin{array}{c}\text { Bastanar/ } \\
\text { Dantewada }\end{array}$ & JK 8 & $\begin{array}{l}\text { Little } \\
\text { millet }\end{array}$ & 1.50 & 5.90 & 2.50 & $\begin{array}{c}3840.0 \\
0\end{array}$ & 0.69 & 69.49 & 4.10 & 3.40 & 136.00 \\
\hline
\end{tabular}


Table.5 Exploitable productivity, technology index, technology gap and extension gap of minor millets as grown under improved practices and farmers practices

\begin{tabular}{|c|c|c|c|c|c|c|c|c|c|}
\hline \multirow{2}{*}{ Crop } & \multirow{2}{*}{ Variety } & \multirow{2}{*}{ Sowing method } & \multirow{2}{*}{$\begin{array}{l}\text { Area } \\
\text { (ha) }\end{array}$} & \multicolumn{2}{|c|}{ yield $\left(q\right.$ ha $\left.^{-1}\right)$} & \multirow{2}{*}{$\begin{array}{c}\% \text { increased } \\
\text { over FP }\end{array}$} & \multirow{2}{*}{$\begin{array}{l}\text { Technology } \\
\text { index }(\%)\end{array}$} & \multirow{2}{*}{$\begin{array}{l}\text { Technology } \\
\operatorname{gap}\left(q \mathbf{h a}^{-1}\right)\end{array}$} & \multirow{2}{*}{$\begin{array}{c}\text { Extension } \\
\operatorname{gap}\left(\mathbf{q} \mathbf{h a}^{-1}\right)\end{array}$} \\
\hline & & & & IP & FP & & & & \\
\hline Finger millet & GPU-28 & Line sowing & 1.11 & 15.09 & 6.33 & 140.12 & 39.63 & 9.91 & 8.76 \\
\hline Kodo millet & JK-439 & Line sowing & 1.01 & 14.57 & 6.29 & 148.64 & 27.17 & 5.43 & 8.28 \\
\hline Little millet & JK-08 & Line sowing & 1.20 & 5.85 & 2.75 & 115.64 & 70.97 & 4.15 & 3.11 \\
\hline
\end{tabular}

Table.6 Economic impact of demonstrated millets as grown under improved practices and farmers practices

\begin{tabular}{|c|c|c|c|c|c|c|c|c|}
\hline \multirow[t]{2}{*}{ Crop } & \multicolumn{2}{|c|}{$\begin{array}{c}\text { Cost of cultivation } \\
\text { (₹ ha-1) }\end{array}$} & \multicolumn{2}{|c|}{$\begin{array}{c}\text { Gross Income } \\
\text { (₹ ha-1) }\end{array}$} & \multicolumn{2}{|c|}{ Net Income } & \multicolumn{2}{|c|}{ B:C Ratio } \\
\hline & IP & FP & IP & FP & IP & FP & IP & FP \\
\hline Finger millet & 8485 & 5300 & 22638 & 11479 & 14153 & 6179 & 1.67 & 1.17 \\
\hline Kodo millet & 6498 & 3600 & 26947 & 11637 & 20449 & 8037 & 3.15 & 2.23 \\
\hline Little millet & 5600 & 3200 & 9366 & 4397 & 3766 & 1197 & 0.67 & 0.37 \\
\hline
\end{tabular}




\section{Technology gap}

The average technology gap in the improved technology was recorded $9.91 \mathrm{q} \mathrm{ha}^{-1}, 5.43 \mathrm{q}$ $\mathrm{ha}^{-1}$ and $4.15 \mathrm{q} \mathrm{ha}^{-1}$ in finger, kodo and little millet (Table 5). It might be due to different climatic and edephic conditions which increase the technology gap. Similar findings were reported by Mukharjee (2003) and Raj et al., (2013).

\section{Technology index}

The technology index shows the feasibility of the evolved technology at the farmer's field and the lower the value of technology index more is the feasibility of the technology (Jeengar, et al., 2006 and Singh, et. al 2007). The index was 39.63, 27.17 and 70.97 percent for the Finger, kodo and little millet (Table 5).

\section{Economic return}

The inputs and outputs prices of produce prevailed during the study of demonstration were taken for calculating cost of cultivation, gross return, net return and benefit: cost ratio (Table 6). The demonstration of small millets (Finger, kodo and little) under improved practices gave higher net return and $\mathrm{B}$ : $\mathrm{C}$ ratio of Rs. 14153.00 and 1.67 for the finger millet, 20449 and 3.15 for kodo millet and 3766 and 0.67 for the little millet. This might be due to higher yield obtained from improved technology as compared to farmer's practices. This finding is also reported by Mokidue et al., 2011 and Raj et al., 2013.

\section{References}

Amadou, I., Gounga M. E. and Le, GuoWei.2013. Millets: Nutritional composition, some health benefits and processing - A Review. Emir. J. Food Agric. 25(7): 501-508.
Anonyms, 1996. Lost crop of Africa. Vol. 1 Grain Board on Science and Technology for International Development. National Research Council National Academy press. Washington D.C., 1996; pp: 171.

Anonyms, 2013-14. Project director review 2013-1: Directorate of maize research, New Delhi

Arun Kumar, M.A., Gali, S.K. and Hebsur, N.S.2007. Effect of different levels of NPK on growth and yield parameters of sweet corn. Karnataka J. Agric. Sci., 20 (1): 41-43.

Burkill, H.M., The useful plants of tropical West Africa (Vol. 2), Royal Botanical Gardens Surrey, U.K, 1985.

Chopra, K., and Neelam M. 2004. Common health problems encountered by the tribal community in Bastar District. Health and Population-Perspectives and Issues, 27 (1): 40-48.

Clayton, W.D. and Renvoze, S.A. 1986. Genera Germanium. Kew Bulletin Additional, 1986; 13, 1-389.

Dhaka, B.L., Meena, B.S and Suwalka R.L. 2010. Popularization of improved maize production technology through frontline demonstrations in South-eastern Rajasthan. Journal of Agricultural Science, 1(1): 39-42.

Dhudhade, D.D., Deshmukh, G, P., Haver, P. N. And Patil, J.V. 2009. Impact of frontline demonstration of chickpea in Maharashtra. Legume Research, 32:206-208.

FAO,

http://faostat.fao.org/site/339/default.as $\mathrm{px}$

Hilu, K.W. and de Wet, J.M.J. 1980. Effect of artificial selection on grain dormancy in Eleusine (Gramineae). Syst. Bot., 5: 5460.

Hiremath, S.M., and Nagaraju, M.V.2010. Evaluation of on-farm frontline demonstrations on the yield of chilli, 
Karnataka Journal of Agricultural Science, 23(2): 341-342.

Jeengar, K.L., Panwar, P. And Pareek, O.P.2006. Front line demonstration on maize in Bhilwada district of Rajasthan. Current Agriculture, 30 (1/2):115-116

Mokidue, I., Mohanty, A.K. and Sanjay, K.2011. Corelating growth, yield and adoption of urd bean technologies. Indian J. Ext. Edu. 11(2):20-24.

Mukherjee, N., 2003. Participatory, learning and action. Concept, publishing Company, New Delhi, pp.63-65.

National Research Council, 1996 . Lost crops of Africa; Volume I Grains. National Academy Press, Washington, DC, 1996.

Painkara, G.P., Salam D.C. and Mishra, R.K.2012. Role of frontline demonstrations on yield enhancement of different crops in Sarguja district of Chhattisgarh. Crop Research. 43(1, 2\&3):298-300.

Poonia, T.C., and Pithia, M.S.2011. Impact of front line demonstrations of chickpea in Gujrat. Legume Research, 34(4):304307.

Pradhan, A., Nag, S. K. and Patil, S.K. 2010. Dietary management of finger millet (Eleusine coracana L. Gaerth) controls diabetes. Current Science, 98(6):763765 .

Raj, A.D., Yadav, V. and Rathod, J.H. 2013.Impact of frontline demonstration (FLD) on the yield of pulses. International Journal of Scientific and Research Publications, 3(9):2013.

Samui, S.K., Maitra, S., Roy, D.K, Mondal,
A.K. and Saha, D. 2000. Evaluation of front line demonstration on groundnut (Arachis hypogeal L.) in Sundarbans. Journal of Indian Society of Costal Agricultural Research, 18 (2):180-183.

Seetharam, A., Riley, K.W. and Harinarayana, G.1986. Small millets in global agriculture. Proceedings of the First International Small Millets Workshop. Bangalore, India, 1986, October 29 -November 2.

Singh, S.N., Singh, V.K., Singh, R.K. and Singh Rakesh K. 2007.Evaluation of on-farm frontline demonstrations on the yield of mustard in Central Plain Zone of Uttar Pradesh. Indian Res. J.Ext.Edu. 7(2\&3):79-81.

Tiwari, K.B., and Saxena, A. 2001. Economic analysis of FLD of oil seeds in Chhindwara. Bhartiya Krishi Anusandhan Patrika, 16 (3\&4):185189.

Van Wyk, B.E., and Gericke, N.2000. People's plants: a guide to useful plants of southern Africa. Arcadia, Pretoria, South Africa: Briza Publications, 2000.

Watson, L. and Dallwitz, M.J. 1992. The grass genera of the world: descriptions, illustrations, identification, and information retrieval; Including synonyms, morphology, anatomy, physiology, phytochemistry, cytology, classification, pathogens, world and local distribution, and references, 1992. Available online: http:// deltaintkey.com/grass. Accessed November, 16 ,

2011.

\section{How to cite this article:}

Ashwani Kumar Thakur, Prafull Kumar and Subhas Chandra Yadav. 2017. Impact of Front Line Demostration (FLD) on the Yield and Economics of Small Millet on Bastar District of Chhattisgarh, India. Int.J.Curr.Microbiol.App.Sci. 6(9): 1489-1497. doi: https://doi.org/10.20546/ijcmas.2017.609.181 